\title{
Humans frequently exposed to a range of non-human primate malaria parasite species through the bites of Anopheles dirus mosquitoes in South-central Vietnam
}

Yoshimasa Maeno ${ }^{1 *}$, Nguyen Tuyen Quang ${ }^{2}$, Richard Culleton ${ }^{3}$, Satoru Kawai ${ }^{4}$, Gaku Masuda ${ }^{5}$, Shusuke Nakazawa ${ }^{6}$ and Ron P. Marchand ${ }^{2}$

\begin{abstract}
Background: Recent studies have described natural human infections of the non-human primate parasites Plasmodium knowlesi and Plasmodium cynomolgi. In Southeast Asia, mosquitoes of the Anopheles leucosphyrus group bite both humans and monkeys in the forest and thus offer a possible route for Plasmodium species to bridge the species barrier. In this study we analysed the species composition of malarial sporozoites infecting the salivary glands of Anopheles dirus in order to determine their potential role as bridge vectors of Plasmodium parasites from monkeys to humans.

Methods: Mosquitoes were collected in the forest and forest fringe area of Khanh Phu commune by human-baited landing collection. Anopheles species were determined on the basis of morphologic features. Sporozoite-infected salivary glands were applied to filter paper and dried in an ambient atmosphere, before storage in closed vials at $4-6{ }^{\circ} \mathrm{C}$. Detection and identification of Plasmodium species in salivary glands were carried out by nested-PCR of the small subunit ribosomal RNA gene.

Results: Six species of Plasmodium parasites were detected by PCR, of which P. vivax was the most common, followed by P. knowlesi, P. inui, P. cynomolgi, P. coatneyi and P. falciparum. Twenty-six of the 79 sporozoite infected mosquitoes showed multiple infections, most of which were a combination of $P$. vivax with one or more of the non-human primate Plasmodium species.

Conclusions: These results suggest that humans overnighting in this forest are frequently inoculated with both human and non-human primate malaria parasites, leading to a situation conducive for the emergence of novel zoonotic malaria.
\end{abstract}

Keywords: Sporozoites, Anopheles dirus, Plasmodium vivax, Plasmodium falciparum, Plasmodium knowlesi, Plasmodium cynomolgi, Plasmodium coatneyi, Plasmodium inui

\footnotetext{
* Correspondence: ymaeno@fujita-hu.ac.jp

${ }^{1}$ Department of Virology and Parasitology, Fujita Health University School of

Medicine, 1-98 Kutsukake, Toyoake, Aichi 470-1192, Japan

Full list of author information is available at the end of the article
} 


\section{Background}

Six malaria parasite species, Plasmodium falciparum, Plasmodium vivax, Plasmodium malariae, Plasmodium ovale wallikeri, Plasmodium ovale curtisi and Plasmodium knowlesi cause disease in humans. In Southeast Asia 13 species of Plasmodium parasites are found in non-human primates [1]. One of these, P. knowlesi, is now a well-known threat to human health in multiple countries in the region [2-6]. Recently, the first naturally acquired human infection of Plasmodium cynomolgi was described from the east coast of Peninsular Malaysia [7]. Little is known regarding the ability of other non-human primate Plasmodium parasites, besides P. knowlesi and P. cynomolgi, to infect humans, though Plasmodium inui, Plasmodium eylesi, Plasmodium schwetzi and others have been recorded as possessing the ability to infect humans as a result of experimental infection [8-16].

Malaria parasites have the ability to switch hosts [17], indeed, it is now thought that of the six species that commonly infect humans, at least three, P. falciparum, $P$. vivax and $P$. knowlesi were originally parasites of nonhuman primates that jumped the species barrier to man.

Zoonotic malaria infections can only occur when the vectors of non-human malaria parasites come into contact with people. Members of the Anopheles dirus complex are known to be important vectors of human Plasmodium parasites in the forests of Southeast Asia, and An. dirus (Species A) has previously been shown to vector $P$. knowlesi in Vietnam $[6,18,19]$. In order to assess the risk of potential zoonotic infections with nonhuman primate parasites, we assayed the malaria parasite species composition of sporozoites residing in the salivary glands of forest-caught $A n$. dirus mosquitoes. Here we report the occurrence of $P$. inui, $P$. cynomolgi and Plasmodium coatneyi in the salivary glands of humanbiting An. dirus mosquitoes in Vietnam.

\section{Methods \\ Parasites}

Positive control genomic DNA (gDNA) for P. falciparum was obtained from 3D7-9A in vitro cultured parasites, whilst $P$. vivax and $P$. malariae gDNA was extracted from Giemsa's solution stained blood films [20]. P. knowlesi $\mathrm{H}$ strain (ATCC No. 30158) gDNA, obtained from an experimentally infected Japanese macaque (Macaca fuscata) [21] was used as a positive control. Twenty-five $\mu \mathrm{L}$ aliquots of clone 3D7-9A or P. knowlesi $\mathrm{H}$ strain infected blood was placed in a micro-reaction tube and kept at $-80{ }^{\circ} \mathrm{C}$ until PCR analysis. The gDNA from $P$. cynomolgi B strain (ATCC No.30129), P. inui Taiwan II strain (ATCC No.30200) and $P$. coatneyi CDC strain was used as a positive control, respectively. These parasites were maintained in Japanese macaques in Dokkyo Medical University and cryopreserved in liquid nitrogen. Throughout the course of the animal experiments, investigators adhered to the Guidelines for the Use of Experimental Animals authorized by the Japanese Association for Laboratory Animal Science. The protocol was approved by the Committee on the Ethics of Animal Experiments of Dokkyo Medical University (Permit Number: 0656).

\section{Mosquito collection and dissection of salivary glands}

Mosquitoes were collected by human-baited landing catches in the forest and forest fringe areas near Nga Hai village in the southern part of Khanh Phu commune, Khanh Vinh district, Khanh Hoa province, Vietnam. Khanh Phu is a commune with around 3,000 inhabitants, mainly of the Raglai ethnic minority, who live between the forested foothills on the east side of the Truong Son mountain range in south central Vietnam, an area where malaria was previously hyper- to holoendemic.

Mosquitoes were collected in the forest south of Khanh Phu $\left(12^{\circ} 11^{\prime} \mathrm{N} ; 108^{\circ} 55^{\prime} \mathrm{E}\right)$ at a place where local people regularly enter the forest, from February 2010 to April 2013. Mosquito collectors were adult men of the Raglai ethnic group. They were regularly screened for malaria and promptly treated with artemisinin combination therapy if infected. The collectors worked in teams of two over the whole night, one person collecting from 18:00 to $24: 00 \mathrm{~h}$ and the other from 0:01 to $6: 00 \mathrm{~h}$. The monthly average collection effort ranged between 18 and 48 person-nights per month, a total of 1,617 personnights over the 36 month period (Table 1).

Anopheles species were determined on the basis of morphology [22]. All An. dirus group mosquitoes were assumed to be $A n$. dirus species A on the basis of previous accurate identifications and the known distribution of this species [23, 24]. Furthermore, a subset of 20 mosquitoes were analysed by PCR and sequencing, and all were shown to be of An. dirus species A (Additional file 1: Figure S1). Female anopheline mosquitoes were dissected for salivary glands, midguts and ovaries and these were examined by microscopy for sporozoites, oocysts and parity, respectively. Sporozoite-infected salivary glands were applied to filter paper and dried in an ambient atmosphere, before storage in closed vials at $4-6{ }^{\circ} \mathrm{C}$.

\section{gDNA extraction and PCR detection}

Extraction of gDNA from sporozoite-infected salivary glands on filter paper and subsequent PCR analysis was carried out as previously described [6, 18]. Briefly, gDNA was extracted using QIAamp DNA Micro Kit (Qiagen, Tokyo, Japan). Plasmodium species-specific nested-PCR assays to identify human and simian Plasmodium species were performed as previously described $[2,25,26]$. The genus-specific primers, rPLU-1/ rPLU-5, were used in the primary amplification (nest 1 ) 
Table 1 Results of the collection, dissections and PCR processing of Anopheles dirus mosquitoes caught by human landing catch in "the forest" and "forest fringe"

\begin{tabular}{|c|c|c|c|c|c|c|c|}
\hline \multicolumn{8}{|c|}{ An. dirus caught in the study area } \\
\hline Year & Period & No. caught & No. nights & Biting density* & No. dissected & No. sporozoites & $\%$ \\
\hline 2010 & Feb to Dec & 2614 & 507 & 5.2 & 2613 & 48 & 1.8 \\
\hline 2011 & Jan to Dec & 1584 & 549 & 2.9 & 1583 & 21 & 1.3 \\
\hline 2012 & Jan to Nov & 1757 & 525 & 3.4 & 1757 & 16 & 0.9 \\
\hline 2013 & Mar to Apr & 116 & 36 & 3.2 & 109 & 1 & 0.9 \\
\hline \multicolumn{2}{|c|}{ Feb/2010 to Apr/2013 } & 6071 & 1617 & 3.8 & 6062 & 86 & 1.4 \\
\hline \multicolumn{8}{|c|}{ An. dirus caught in the forest } \\
\hline 2010 & Feb to Dec & 1668 & 303 & 5.5 & 1667 & 33 & 2 \\
\hline 2011 & Jan to Dec & 1093 & 329 & 3.3 & 1092 & 15 & 1.4 \\
\hline 2012 & Jan to Nov & 920 & 283 & 3.3 & 920 & 9 & 1.0 \\
\hline 2013 & Mar to Apr & 27 & 10 & 2.7 & 26 & 1 & 3.8 \\
\hline \multicolumn{2}{|c|}{ Feb/2010 to Apr/2013 } & 3708 & 925 & 4.0 & 3705 & 58 & 1.6 \\
\hline \multicolumn{8}{|c|}{ An. dirus caught in forest fringe } \\
\hline 2010 & Feb to Dec & 946 & 204 & 4.6 & 946 & 15 & 1.6 \\
\hline 2011 & Jan to Dec & 491 & 220 & 2.2 & 491 & 6 & 1.2 \\
\hline 2012 & Jan to Nov & 837 & 242 & 3.5 & 837 & 7 & 0.8 \\
\hline 2013 & Mar to Apr & 89 & 26 & 3.4 & 83 & 0 & 0.0 \\
\hline \multicolumn{2}{|c|}{ Feb/2010 to Apr/2013 } & 2363 & 692 & 3.4 & 2357 & 28 & 1.2 \\
\hline
\end{tabular}

*Biting density, average human-biting density (No. of caught / No. of caught person-night)

and performed as described by Singh et al. (1999) [25]. Detection of species-specific 18S rRNA genes (nest 2) was performed as previously described $[2,25,26]$. For the nest $2,2 \mu \mathrm{L}$ of $50 \times$ nest 1 amplification product was used as the template in the reaction mixtures $(25 \mu \mathrm{L})$. For authenticating $P$. knowlesi infection, detection of the circumsporozoite protein (CSP) gene of $P$. knowlesi from samples was carried out as previously described by Vythilingam et al. (2008) [27]. A 2720 Thermal cycler (ABI, Foster city, CA, USA) was used for all PCRs. PCR products were separated by electrophoresis on $1.5 \%$ agarose gels and stained with ethidium bromide. DNA bands were analysed with Lane \& Spot Analyzer software (Atto, Tokyo, Japan). No amplification was observed with mosquito gDNA controls. Primer sequences for 18SrRNA of human and non-human primate Plasmodium species-specific primers $[2,25,26]$, and the CSP gene of P. knowlesi [27] were as previously described.

\section{Sequencing and analysis of DNA for 18SrRNA of monkey Plasmodium species}

For nucleotide sequencing, the specific products resulting from PCR amplification of the $18 S$ rRNA of non-human primate Plasmodium species were cleaned using the Wizard SV Gel and PCR Clean-up System (Promega, Tokyo, Japan) according to the manufacturer's instructions, and were then sequenced with the BigDye Terminator v3.1 Cycle Sequencing Premix Kit (ABI). The reaction products for sequencing were separated with an ABI/Hitachi 3130x1 Genetic Analyzer (ABI) and the resulting nucleotide sequences were compiled using Genetyx (Genetyx Corporation, Tokyo, Japan). A phylogenetic tree constructed using these sequences is shown in Additional file 2: Figure S2.

\section{Statistical analysis}

Statistical evaluation was performed using with the $X^{2}$-test or the Mann-Whitney test (two-tailed). All analyses were performed using SPSS software (SPSS Japan, Tokyo, Japan), values of $P<0.05$ was considered significant.

\section{Results}

Plasmodium parasite infections in An. dirus mosquitoes

During this period, as well as over many years before in this forest, An. dirus was the only anopheline species found with sporozoites. An. dirus mosquitoes were found during every month of sampling with an average human-biting density of 3.8 bites/person-night (range 2.9-5.2) in this study period. This human-biting density 
did not show significant differences between mosquito collection sites (Table 1 ).

A total of 6,071 female An. dirus were captured of which 6,062 were dissected for the detection of sporozoites in salivary glands by microscopic examination and 86 (1.4 \%) were infected with Plasmodium sporozoites. Of the mosquitoes infected with sporozoites, 58 out of 3,705 (1.6\%) were found in the forest and 28 out of $2,357(1.2 \%)$ in the forest fringe. Both the biting densities as well as the infection rates were not significantly different between the sites of collection (Table 1).

\section{Identification of Plasmodium species in mosquito salivary glands}

Of the 86 sporozoite-positive mosquitoes, 83 underwent nested PCR analysis for identification of species of Plasmodium sporozoites, of which 79 were successfully assayed. All of the PCR positive sporozoite samples were identified to the Plasmodium species level.

It has been shown previously that the $18 s R N A$ primer set specific for $P$. knowlesi can occasionally cross-react with $P$. vivax genomic DNA [28]. Therefore, identification of $P$. knowlesi was determined by three different PCR assays, and confirmed by sequencing of the products. Seventeen out of 79 PCR-positive samples were confirmed as $P$. knowlesi through sequencing of the $18 \mathrm{~S}$ rRNA gene.

\section{Prevalence of Plasmodium species infections}

Using a nested PCR assay, we detected six species of Plasmodium parasites, with $P$. vivax being most common (prevalence of $43 \%$ ), followed by $P$. knowlesi (15\%), P. inui (15\%), P. cynomolgi (10 \%), P. coatneyi (9 \%) and P. falciparum (8 \%). Plasmodium malariae and $P$. ovale were not detected (Table 2).

Fifty-three out of 79 mosquitoes were positive for single Plasmodium species. Among them, $P$. vivax was dominant (prevalence of $51 \%$ ), followed by $P$. coatneyi (13\%), P. cynomolgi (11 \%), P. inui (9 \%), P. falciparum (8\%) and P. knowlesi (8\%) (Table 2). Co-infections of Plasmodium species were common, with 26 of the 79 (33\%) mosquitoes being infected by two or more species of Plasmodium each, most of the cases being mixed infection with $P$. vivax and another species (Fig. 1, Table 2). As shown in Table 2, P. vivax coinfections with the non-human primate Plasmodium species, P. knowlesi (33\%), P. inui (27 \%), P. cynomolgi $(17 \%)$ and P. coatneyi (7 \%), were observed. For P. falciparum, co-infection with non-human primate Plasmodium species was observed only with $P$. inui (7 \%). In contrast, co-infection among human Plasmodium species was observed in only two cases, with $P$. vivax and $P$. falciparum (10 \%) (Table 2).
Table 2 Summary of Plasmodium spp. infections in Anopheles dirus

\begin{tabular}{|c|c|c|}
\hline Infection & Plasmodium spp. & No. of mosquitoes infected \\
\hline \multirow[t]{6}{*}{ Single (53) } & Pf & 4 \\
\hline & $\mathrm{PV}$ & 27 \\
\hline & Pk & 4 \\
\hline & Pct & 7 \\
\hline & Pcy & 6 \\
\hline & Pin & 5 \\
\hline \multirow[t]{6}{*}{ Double (19) } & $P f, P v$ & 3 \\
\hline & Pf, Pin & 1 \\
\hline & $\mathrm{PV}, \mathrm{Pk}$ & 7 \\
\hline & Pv, Pcy & 3 \\
\hline & Pv, Pin & 3 \\
\hline & Pk, Pin & 2 \\
\hline \multirow[t]{5}{*}{ Triple (6) } & Pf, Pv, Pin & 1 \\
\hline & $P \vee, P k, P c y$ & 1 \\
\hline & Pv, Pk, Pin & 2 \\
\hline & Pv, Pct, Pin & 1 \\
\hline & Pk, Pct, Pin & 1 \\
\hline Quadruple & Pv, Pct, Pcy, Pin & 1 \\
\hline \multicolumn{2}{|c|}{ No. of PCR-positive } & 79 \\
\hline \multicolumn{2}{|c|}{ No. of PCR-negative } & 4 \\
\hline \multicolumn{2}{|c|}{ Total no. of examined sporozoite-positive } & 83 \\
\hline
\end{tabular}

$\mathrm{Pf}=P$. falciparum, $\mathrm{Pv}=P$. vivax, $\mathrm{Pk}=P$. knowlesi, $\mathrm{Pct}=P$. coatneyi, $\mathrm{Pcy}=P$. cynomolgi, Pin $=P$. inui

\section{Comparison of Plasmodium species infections by collection site and biting time}

Although the data show a tendency towards more mosquitoes being infected with $P$. cynomolgi or $P$. inui in the forest compared with the forest fringe (Table 3), none of the differences between collection sites were significant. Anopheles dirus started to bite humans from right after sunset with a peak from 20:00 to 22:00 h and with a second peak from 0:00 to 2:00 h (Fig. 2). There were no significant differences in biting times between the forest fringe and the forest collection sites, or in the Plasmodium species composition at different times of the night.

\section{Discussion}

We evaluated the transmission of Plasmodium parasites by An. dirus in a forested area in the Khanh Phu commune, Vietnam, where previous studies demonstrated their co-infection with $P$. knowlesi, P. falciparum, $P$. vivax and $P$. malariae. $[6,18]$. Our results suggest that both humans and macaques are commonly bitten by $A n$. dirus in the forests around the Khanh Phu commune. To obtain data to verify whether and which macaques are the reservoirs of non-human primate Plasmodium 


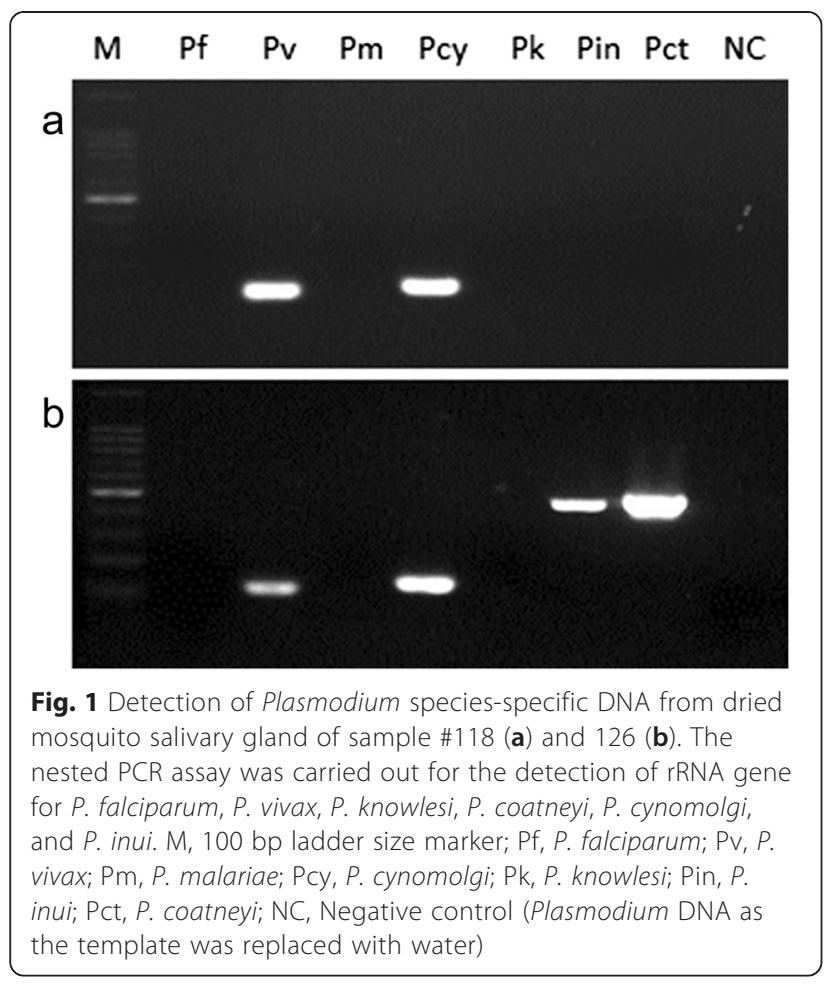

Table 3 Comparison of Plasmodium spp. infections in Anopheles dirus by the collection sites

\begin{tabular}{|c|c|c|c|}
\hline \multirow[t]{2}{*}{ Infection } & \multirow[t]{2}{*}{ Plasmodium spp. } & \multicolumn{2}{|c|}{ No. of mosquitoes infected } \\
\hline & & Forest fringe & In the forest \\
\hline \multirow[t]{6}{*}{ Single } & Pf & 1 & 3 \\
\hline & $\mathrm{PV}$ & 10 & 17 \\
\hline & $\mathrm{Pk}$ & 1 & 3 \\
\hline & Pct & 4 & 3 \\
\hline & Pcy & 0 & 6 \\
\hline & Pin & 1 & 4 \\
\hline \multirow[t]{6}{*}{ Double } & $\mathrm{Pf}, \mathrm{PV}$ & 1 & 2 \\
\hline & Pf, Pin & 0 & 1 \\
\hline & $\mathrm{PV}, \mathrm{Pk}$ & 3 & 4 \\
\hline & Pv, Pcy & 0 & 3 \\
\hline & PV, Pin & 2 & 1 \\
\hline & Pk, Pin & 0 & 2 \\
\hline \multirow[t]{5}{*}{ Triple } & Pf, Pv, Pin & 1 & 0 \\
\hline & $\mathrm{PV}, \mathrm{Pk}, \mathrm{Pcy}$ & 0 & 1 \\
\hline & $\mathrm{PV}, \mathrm{Pk}$, Pin & 0 & 2 \\
\hline & Pv, Pct, Pin & 1 & 0 \\
\hline & Pk, Pct, Pin & 0 & 1 \\
\hline Quadruple & Pv, Pct, Pcy, Pin & 1 & 0 \\
\hline \multicolumn{2}{|c|}{ No.of examined sporozoite-positive } & 26 & 53 \\
\hline
\end{tabular}

$\mathrm{Pf}=P$. falciparum, $\mathrm{Pv}=P$. vivax, $\mathrm{Pk}=P$. knowlesi, $\mathrm{Pct}=P$. coatneyi, $\mathrm{Pcy}=P$. cynomolgi, $\mathrm{Pin}=P$. inui parasites we previously collected and analysed macaque faecal samples collected from the forest floor, and from captive macaques in a cage in the forest where An. dirus are commonly found. Caged macaques were infected with $P$. cynomolgi, P. coatneyi, P. inui and P. knowlesi, with the latter species detectable only in faecal samples [29]. The data presented here show that An. dirus in the Khanh Phu forest are infected with two species of human Plasmodium parasites, P. falciparum and P. vivax, and four species of non-human primate Plasmodium parasites, P. knowlesi, P. inui, P. cynomolgi and P. coatneyi. Approximately one-third of mosquitoes were infected by two or more species of Plasmodium, and these were often a combination of human and non-human primate parasites. The numerous co-infections and the lack of differences between collection sites and biting times in addition suggest that all these parasites are transmitted by just one population of An. dirus, which bite humans as readily as macaques.

Among the non-human primate Plasmodium parasite sporozoites identified in this study, P. knowlesi dominated, followed by $P$. inui, P. cynomolgi and P. coatneyi. Several studies in Southeast Asia have demonstrated similar prevalences of non-human primate Plasmodium species in macaques. In Singapore, P. knowlesi was the most common species followed by $P$. cynomolgi and $P$. inui [30]. In Malaysian Borneo, however, the dominant species was $P$. inui followed by $P$. knowlesi, $P$. coatneyi and $P$. cynomolgi [26].

The results of this study indicate that humans overnighting in Khanh Phu forest are frequently inoculated with a range of simian Plasmodium parasites, several of which have been shown to be capable of causing disease in humans $[2-8,11,14,15,31]$. The implications of this situation are potentially serious. As it is known that malaria parasites are capable of jumping species and causing

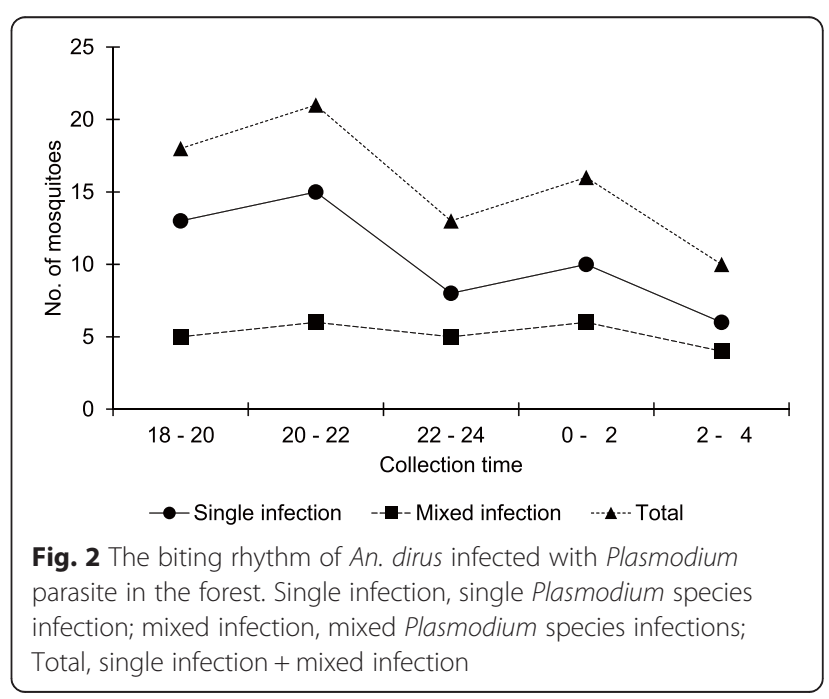


zoonotic infections in certain circumstances, the major factor governing the chances of a new zoonotic species emerging is exposure. The greater the number of inoculations into humans of macaque parasites, and the greater the number and diversity of species being introduced, the higher the chance becomes of either a permissible human host being encountered, or the inoculation of a mutant parasite capable of causing a zoonotic infection. Situations such as the one identified here, in which humans are routinely and regularly exposed to inoculations of (currently) non-human primate sporozoites, are likely to be highly conducive to the emergence of novel zoonotic malaria infections, and should be prevented as much as possible and monitored closely.

\section{Conclusion}

We report the detection of sporozoites of both human and non-human primate Plasmodium parasites from the same mosquito population. Six species of Plasmodium were detected by PCR, of which $P$. vivax was the most common, followed by P. knowlesi, P. inui, P. cynomolgi, $P$. coatneyi and P. falciparum. About one-third of sporozoite infected mosquitoes showed multiple infections, most of which were a combination of $P$. vivax with one or more of the non-human primate Plasmodium species. These results suggest that humans overnighting in this forest are frequently inoculated with these malaria parasites, leading to a situation conducive for the emergence of novel zoonotic malaria parasites.

\section{Additional files}

Additional file 1: Figure S1. Phylogenetic tree showing how the mosquitoes considered in this work relate to other members of the Anopheles dirus complex. We amplified and sequences a bp fragments of ribosomal DNA internal transcribed spacer 2 gene from a subset of 20 mosquitoes identified as Anopheles dirus species A. As a result of BLAST analysis, sequence of KP samples showed $99 \%$ homology to An. dirus A (U60410), and in contrast, only $97 \%$ homology to An. dirus D. The evolutionary history was inferred using the Neighbor-Joining method. The percentage of replicate trees in which the associated taxa clustered together in the bootstrap test (1000 replicates) are shown next to the branches. The tree is drawn to scale, with branch lengths in the same units as those of the evolutionary distances used to infer the phylogenetic tree. The evolutionary distances were computed using the Kimura 2-parameter method and are in the units of the number of base substitutions per site. Evolutionary analyses were conducted in MEGA5.

Additional file 2: Figure S2. Phylogenetic tree based on the sequence of the 18sSSUrRNA gene of malaria parasites isolated from mosquitoes. The evolutionary history was inferred using the Neighbor-Joining method. The percentage of replicate trees in which the associated taxa clustered together in the bootstrap test (1000 replicates) are shown next to the branches. The tree is drawn to scale, with branch lengths in the same units as those of the evolutionary distances used to infer the phylogenetic tree. The evolutionary distances were computed using the Kimura 2-parameter method and are in the units of the number of base substitutions per site. Evolutionary analyses were conducted in MEGA5. By the BLAST analysis, sequences of $P$. inui and $P$. coatneyi was asexual stage (A type) and that of $P$. cynomolgi and $P$. knowlesi was sexual stage (S type).

\section{Competing interests}

The authors declare no competing interests.

\section{Authors' contributions}

Conceived and designed the study: YM, NTQ, SN and RPM. Performed the field work and data collection: NTQ, GM and RPM. Performed the experiments: YM, SK, SN and RC. Performed statistical analysis: YM, NTQ, GM and RPM. Wrote the paper: YM, SK, SN, RC, NTQ, RPM and GM. All authors read and approved the final manuscript.

\section{Acknowledgments}

We thank Nguyen Son Hai, Phan Chau Do, Dang Duy Vu and Nguyen Le Dung for their technical support. We also thank the Provincial Health Service and Malaria Control Centre of Khanh Hoa Province as well as the authorities of Khanh Vinh district and Khanh Phu municipality for their administrative support.

This study was supported in part by the following five grants: (i) the Japan Society for the Promotion of Science (JSPS) Asia Africa Science Platform Program, (ii) the Grant-in-Aid for Scientific Research No. 23406022 and 26360029 from the Japan Society for the Promotion of Science, (iii) the Cooperative Research Grant (2011-A-7, 2012-A-5) of the Institute of Tropical Medicine, Nagasaki University, (iv) a Grant-in-Aid for Scientific Research from Fujita Health University, (v) the Center for Infectious Disease Research in Asia and Africa: Vietnam Research Station, Nagasaki University, and the Program of Japan Initiative for Global Research Network on Infectious Diseases (J-GRID), MEXT, Japan. The Khanh Phu Malaria Research team and the field research costs were financially supported through private donations to the Medical Committee NetherlandsVietnam.

\section{Author details}

${ }^{1}$ Department of Virology and Parasitology, Fujita Health University School of Medicine, 1-98 Kutsukake, Toyoake, Aichi 470-1192, Japan. ${ }^{2}$ Khanh Phu Malaria Research Unit, Medical Committee Netherlands-Viet Nam, Nha Trang, Khanh Hoa province, Viet Nam. ${ }^{3}$ Malaria Unit, Institute of Tropical Medicine, Nagasaki University, Nagasaki, Nagasaki, Japan. ${ }^{4}$ Laboratory of Tropical Medicine and Parasitology, Dokkyo Medical University, Mibu, Tochigi, Japan. ${ }^{5}$ The Graduate School of Global Environmental Studies, Kyoto University, Kyoto, Kyoto, Japan. ${ }^{6}$ Department of Protozoology, Institute of Tropical Medicine, Nagasaki University, Nagasaki, Nagasaki, Japan.

Received: 10 April 2015 Accepted: 9 July 2015

Published online: 16 July 2015

\section{References}

1. Collins WE. Major animal models in malaria research: Simian. In: Wernsdofer WH, McGregor, editors. Malaria: Principles and Practice of Malariology. Edinburgh: Churchill Livingstone; 1988. p. 1473-501.

2. Singh B, Kim Sung L, Matusop A, Radhakrishnan A, Shamsul SS, Cox-Singh J, et al. A large focus of naturally acquired Plasmodium knowlesi infections in human beings. Lancet. 2004;363:1017-24.

3. Putaporntip C, Hongsrimuang T, Seethamchai S, Kobasa T, Limkittikul K, Cui L, et al. Differential prevalence of Plasmodium infections and cryptic Plasmodium knowlesi malaria in humans in Thailand. J Infect Dis. 2009;199:1143-50. doi:10.1086/597414.

4. Luchavez J, Espino F, Curameng P, Espina R, Bell D, Chiodini P, et al. Human Infections with Plasmodium knowlesi, the Philippines. Emerg Infect Dis. 2008;14:811-3. doi:10.3201/eid1405.071407.

5. Jiang N, Chang Q, Sun X, Lu H, Yin J, Zhang Z, et al. Co-infections with Plasmodium knowlesi and other malaria parasites, Myanmar. Emerg Infect Dis. 2010;16:1476-8. doi:10.3201/eid1609.100339.

6. Marchand RP, Culleton R, Maeno Y, Quang NT, Nakazawa S. Co-infections of Plasmodium knowlesi, P. falciparum, and P. vivax among humans and Anopheles dirus mosquitoes, Southern Vietnam. Emerg Infect Dis. 2011;17:1232-9. doi:10.3201/eid1707.101551.

7. Ta TH, Hisam S, Lanza M, Jiram Al, Ismail N, Rubio JM. First case of a naturally acquired human infection with Plasmodium cynomolgi. Malar J. 2014;13:68. doi:10.1186/1475-2875-13-68 
8. Schmidt LH, Greenland R, Genther CS. The transmission of Plasmodium cynomolgi to man. Am J Trop Med Hyg. 1961;10:679-88.

9. Deane LM, Deane MP, Ferreira NJ. Studies on transmission of simian malaria and on a natural infection of man with Plasmodium simium in Brazil. Bul World Health Organ. 1966;35:805-8.

10. Coatney GR, Chin W, Contacos PG, King HK. Plasmodium inui, a quartan-type malaria parasite of Old World monkeys transmissible to man. J Parasitol. 1966;52:660-3.

11. Coatney GR, Colins WE, Warren M, Contacos PG. The Primate Malaria. Washington, D.C.: U.S. Government Print. Office; 1971. p. 43-339. Stock No. 1744-0005.

12. Chin W, Contacos PG, Collins WE, Jeter MH, Alpert E. Experimental mosquito transmission of Plasmodium knowlesi to man and monkey. Am J Trop Med Hyg. 1968;17:355-8.

13. Contacos PG, Coatney GR, Orihel TC, Collins WE, Chin W, Jeter MH. Transmission of Plasmodium schwetzi from the chimpanzee to man by mosquito bite. Am J Trop Med Hyg. 1970;19:190-5.

14. Cross JH, Hsu-Kuo MY, Lien JC. Accidental human infection with Plasmodium cynomolgi bastianellii. Southeast Asian J Trop Med Public Health. 1973;4:481-3

15. Most H. Plasmodium cynomolgi malaria: accidental human infection. Am J Trop Med Hyg. 1973;22:157-8.

16. Baird JK. Malaria zoonoses. Travel Med Infect Dis. 2009:7:269-77.

17. Hayakawa T, Arisue N, Udono T, Hirai H, Sattabongkot J, Toyama T, et al. Identification of Plasmodium malariae, a human malaria parasite, in imported chimpanzees. PLoS One. 2009;4(10):e7412. doi:10.1371/ journal.pone.0007412.

18. Nakazawa S, Marchand RP, Quang NT, Culleton R, Manh ND, Maeno Y. Anopheles dirus co-infection with human and monkey malaria parasites in Vietnam. Int J Parasitol. 2009;39:1533-7. doi:10.1016/j.ijpara.2009.08.005.

19. Hii J, Rueda LM. Malaria vectors in the Greater Mekong Subregion: overview of malaria vectors and remaining challenges. Southeast Asian J Trop Med Public Health. 2013;44 Suppl 1:73-165. discussion 306-307.

20. Maeno Y, Nakazawa S, le Dao D, Yamamoto N, Giang ND, Van Hanh T, et al. A dried blood sample on filter paper is suitable for detecting Plasmodium falciparum gametocytes by reverse transcription polymerase chain reaction. Acta Trop. 2008;107:121-7. doi:10.1016/j.actatropica.2008.05.001.

21. Kawai S, Hirai M, Haruki K, Tanabe K, Chigusa Y. Cross-reactivity in rapid diagnostic tests between human malaria and zoonotic simian malaria parasite Plasmodium knowlesi infections. Parasitol Int. 2009;58:300-2. doi:10.1016/j.parint.2009.06.004.

22. Vietnam National Institute of Malariology, Parasitology and Entomology. Identifi cation key for Anophelinae in Vietnam. Hanoi (Vietnam): Medical Publishing House; 2008.

23. Trung HD, Van Bortel W, Sochantha T, Keokenchanh K, Quang NT, Cong LD, et al. Malaria transmission and major malaria vectors in different geographical areas of Southeast Asia. Trop Med Int Health. 2004;9:230-7.

24. Obsomer V, Defourny P, Coosemans M. The Anopheles dirus complex: spatial distribution and environmental drivers. Malar J. 2007;6:26.

25. Singh B, Bobogare A, Cox-Singh J, Snounou G, Abdullah MS, Rahman HA. A genus- and species-specific nested polymerase chain reaction malaria detection assay for epidemiologic studies. Am J Trop Med Hyg. 1999;60:687-92.

26. Lee KS, Divis PC, Zakaria SK, Matusop A, Julin RA, Conway DJ, et al. Plasmodium knowlesi: reservoir hosts and tracking the emergence in humans and macaques. PLoS Pathog. 2011;7(4):e1002015. doi:10.1371/ journal.ppat.1002015

27. Vythilingam I, Noorazian YM, Huat TC, Jiram Al, Yusri YM, Azahari AH, et al. Plasmodium knowlesi in humans, macaques and mosquitoes in peninsular Malaysia. Parasit Vectors. 2008;1:26. doi:10.1186/1756-3305-1-26.

28. Imwong M, Tanomsing N, Pukrittayakamee S, Day NP, White NJ, Snounou G. Spurious amplification of a Plasmodium vivax small-subunit RNA gene by use of primers currently used to detect $P$. knowlesi. J Clin Microbiol. 2009;47:4173-5. doi:10.1128/JCM.00811-09.

29. Abkallo HM, Liu W, Hokama S, Ferreira PE, Nakazawa S, Maeno Y, et al. DNA from pre-erythrocytic stage malaria parasites is detectable by PCR in the faeces and blood of hosts. Int J Parasitol. 2014;44(7):467-73. doi:10.1016/ j.ijpara.2014.03.002

30. Li M. Identification and molecular characterization of simian malaria parasites in wild monkeys of Singapore. Singapore: Natinal University of Singapore; 2011

31. Garnham PCC, Molinari V, Shute PG. Differential diagnosis of bastianellii and vivax malaria. Bull World Health Organ. 1962;27:199-202.

\section{Submit your next manuscript to BioMed Central and take full advantage of:}

- Convenient online submission

- Thorough peer review

- No space constraints or color figure charges

- Immediate publication on acceptance

- Inclusion in PubMed, CAS, Scopus and Google Scholar

- Research which is freely available for redistribution

Submit your manuscript at www.biomedcentral.com/submit 